\title{
Vacuna contra fiebre amarilla para viajeros chilenos a Brasil. Consideraciones prácticas
}

\author{
Thomas Weitzel', Cecilia Perret2,3, Francisca Valdivieso', Katia Abarca y Pablo Vial' \\ Yellow fever vaccination for Chilean tourists visiting Brazil. \\ Practical considerations
}

Due to the recent yellow fever outbreak affecting the costal region of Brazil, including main touristic destinations, there is a high demand of yellow fever vaccination. This publication addresses the most relevant practical issues regarding this vaccine for tourists visiting Brazil and aims to serve as a guideline for non-expert physicians in Chile and elsewhere.

Keywords: Yellow fever, vaccine, Travel Medicine, Brazil, outbreak.

Palabras clave: Fiebre amarilla, vacuna, Medicina del Viajero, Brasil, brote.

$\mathrm{B}$ rasil es uno de los principales destinos turísticos para chilenos, con más de 280.000 viajeros registrados en 2017. La reciente introducción de la fiebre amarilla (FA) en las regiones costeras de ese país, desde el Estado de Bahía hacia el sur, incluyendo sus principales ciudades turísticas, constituye un desafío para los médicos que trabajan en Medicina del Viajero y para los centros donde se administra la vacuna en Chile. La amenaza de la FA para viajeros no es solo teórica, como quedó demostrado por los recientes casos en viajeros no vacunados, que incluyeron dos casos fatales en chilenos que visitaron la localidad de Isla Grande ${ }^{1}$. En general, se aconseja a los visitantes a regiones endémicas de FA que reciban la vacuna con anticipación; esto es aún más importante para visitas a regiones costeras de Brasil recientemente afectadas que poseen un elevado e impredecible riesgo de presentar una situación de brote epidémico.

La pregunta de qué viajero necesita la vacuna contra la FA es un tópico clásico en Medicina del Viajero, requiriendo para su respuesta de información detallada de la distribución global de FA y los requisitos de entrada de cada país visitado. La vacuna contra la FA tiene por objetivo prevenir que los viajeros presenten manifestaciones graves de FA, las que tienen una muy elevada tasa de letalidad. De los 10 casos de FA relacionados a viajes reportados en la literatura médica entre 1979 y 2002, sólo uno sobrevivió ${ }^{2}$. La administración de la vacuna contra la FA es compleja por dos aspectos; primero: debido a la necesidad de una estricta cadena de frío y aspectos legales relacionados a la emisión del certificado de vacunación, sólo un número limitado de vacunatorios esta autorizados para administrar la vacuna y segundo: la vacuna contra la FA es una vacuna viva atenuada, con riesgo de efectos adversos serios, que por tanto, requiere una evaluación riesgo-beneficio de cada persona que viaja a regiones endémicas o países que tienen como requisito de entrada la certificación de la vacunación ${ }^{3}$. Como un problema adicional, la capacidad de producción de la vacuna contra la FA es limitada, a causa de la complejidad de su fabricación.

Debido al número creciente de brotes epidémicos, a la diseminación del virus en nuevas áreas geográficas, y al aumento del número de personas expuestas e infectadas en las últimas dos décadas, la escasez o falta de disponibilidad de la vacuna contra la FA es un problema emergente en varios países incluyendo Chile, que muy probablemente persistirá en el futuro próximo.

El brote de FA en curso en Brasil ha provocado un masivo aumento de personas que requieren de la vacunación en Chile. Muchos de estos son turistas que visitan la costa de Brasil, a menudo con sus familias y la mayoría por un período bastante corto de tiempo. Sao Paulo y Río de Janeiro son también frecuentes destinos para viajes de trabajo desde Chile. Como consecuencia, muchos médicos sin experiencia en Medicina del Viajero son y serán confrontados con preguntas de pacientes que están planificando visitar Brasil.

Las siguientes recomendaciones resumen algunos de los temas prácticos relevantes para médicos no expertos de Chile y de otros países.

\section{- ¿Cómo deben planificar su vacunación los viajeros a Brasil?}

Debido a que la disponibilidad de la vacuna contra la FA en Chile es limitada e impredecible, las personas que planean viajar a Brasil (o a otros destinos que requieren la vacunación) deben buscar consejería de viaje con bastante anticipación (idealmente varios meses).
'Programa de Medicina del Viajero, Clínica Alemana, Facultad de Medicina Clínica Alemana, Universidad del Desarrollo, Santiago, Chile.

${ }^{2}$ Centro de Medicina del Viajero, Escuela de Medicina, Pontificia Universidad Católica de Chile, Santiago, Chile.

${ }^{3}$ Departamento de Enfermedades Infecciosas e Inmunología Pediátricas, Escuela de Medicina, Pontificia Universidad Católica de Chile, Santiago, Chile. ${ }^{4}$ Instituto de Ciencias e Innovación en Medicina, Facultad de Medicina, Clínica Alemana Universidad del Desarrollo, Santiago, Chile.

Conflictos de interés: ninguno. Financiamiento: ninguno.

Recibido: 16 de octubre de 2018. Aceptado: 21 de octubre de 2018.

Correspondencia a: Thomas Weitzel thomas.weitzel@gmail.com 
- ¿Es obligatoria la vacuna contra la FA para viajar a Brasil?

Brasil no requiere la vacunación contra la FA (ni ninguna otra vacuna) para entrar al país. Sin embargo, para protegerse de la infección, todos los viajeros a áreas afectadas deben ser vacunados. Los viajeros deben seguir, además, las recomendaciones para la prevención de picaduras por mosquitos.

- ¿Qué responder a un viajero que pregunta si la vacuna contra la FA es realmente necesaria?

Los viajeros deben ser informados acerca de la gravedad de la FA, enfermedad que puede manifestarse como una fiebre hemorrágica con una tasa de letalidad comparable con la infección por virus Ébola. La enfermedad es transmitida por mosquitos infectados. Las picaduras por mosquitos son inevitables en regiones tropicales aún si se usan repelentes y otras medidas. El riesgo epidemiológico de infección depende de varios factores y es impredecible, especialmente si la enfermedad ha sido recientemente introducida en una región (como la costa de Brasil). Como consecuencia lógica, cualquier persona que entra a un área de riesgo debería ser vacunada con la debida antelación.

- ¿Cuán efectiva es la vacuna? ¿Pueden los viajeros infectarse a pesar de estar vacunados?

La efectividad precisa de la vacuna contra la FA es desconocida ya que no se han efectuado estudios clínicos controlados. A pesar de eso, basado en datos epidemiológicos y experiencia clínica, la vacuna es considerada una

\begin{tabular}{|c|c|}
\hline Contraindicaciones & $\begin{array}{l}\text { - Edad }<6 \text { meses } \\
\text { - Mujeres dando lactancia a niños }<6 \text { meses de edad o a lactantes con } \\
\text { inmunodeficiencia grave } \\
\text { - Inmunodeficiencia grave* } \\
\text { - Alergia al huevo grave }\end{array}$ \\
\hline Precauciones** & $\begin{array}{l}\text { - Edad entre 6-9 meses } \\
\text { - Mujeres dando lactancia a niños entre 6-9 meses de edad } \\
\text { - Edad > } 60 \text { años } \\
\text { - Embarazo } \\
\text { - Inmunodeficiencia moderada }\end{array}$ \\
\hline
\end{tabular}

*Inmunodeficiencias primarias, enfermedades del timo, infección por VIH sintomática o con valores de linfocitos $\mathrm{T} C D 4+<200$ por $\mu \mathrm{L}$, quimioterapia reciente (para cáncer) o irradación cuyo blanco sean células inmunológicas o trasplante de precursores hematopoyéticos o de órganos sólidos, tratamiento con fármacos inmunodepresores o inmunomoduladores (p. ej.: corticosteroides en dosis altas, fármacos alquilantes, antimetabolitos, inhibidores de FNT, de IL-1, u otros anticuerpos monoclonales dirigidos a células inmunológicas $)^{3}$. ** La vacunación debe hacerse sólo después de una evaluación personalizada de los riesgos y beneficios por un médico con experiencia. de las vacunas más efectivas disponibles. En los últimos 70 años más de 500 millones de personas han sido vacunadas y sólo se han reportado 18 casos de falla de la vacuna ${ }^{4}$.

- ¿Deberían vacunarse los viajeros que visitan áreas de Brasil sin riesgo de FA o que hacen escala en los aeropuertos de Sao Paulo o Río de Janeiro?

Para viajeros que planean visitar exclusivamente regiones en Brasil sin riesgo de FA, la vacuna no está indicada. Lo mismo aplica para viajeros que sólo hacen escala en aeropuertos de Sao Paulo o Río de Janeiro.

\section{- ¿Qué deben considerar los médicos si pres- criben vacuna contra la FA?}

Los médicos que emiten prescripciones para la vacuna contra la FA deben evaluar a cada paciente para posibles contraindicaciones y precauciones, advertir sobre posibles efectos adversos y que deben evitar el embarazo (al menos por 1 mes después de la vacunación). Los viajeros con contraindicaciones no deben recibir la vacuna; aquellos con posibles contraindicaciones o con precauciones (Tabla 1) deben ser evaluados en forma individual por un médico con experiencia en Medicina del Viajero.

\section{- ¿Cuál es el mejor momento para recibir la vacuna contra la $\mathbf{F A}$ ?}

La producción de anticuerpos comienza aproximadamente 5 días después de la administración de la vacuna y alcanza niveles protectores después de 10 días. Por lo tanto, se consideran protegidos aquellos viajeros que reciben la vacuna al menos 10 días antes de entrar a un área de riesgo. Sin embargo, si hay suficiente tiempo, es recomendable recibir la vacuna 4 semanas o más antes del viaje.

- ¿Qué hacer si un viajero no está seguro de haber recibido la vacuna contra la FA anteriormente?

Si no hay registro de la administración y es incierto si recibió la vacuna de FA, el viajero debe recibir la vacuna. Vacunaciones repetidas con cualquier lapso entre dosis no se asocian con mayor riesgo de efectos adversos, al contrario, las revacunaciones tienen un bajo riesgo de efectos secundarios.

- ¿Cuánto tiempo protege la vacuna? ¿Qué viajeros necesitan una revacunación o refuerzo (booster)?

De acuerdo a recientes recomendaciones de la OMS, la vacuna contra la FA otorga protección de por vida y 
el certificado de vacunación es también válido de por $v$ vida $^{3}$. Esta nueva guía incluye la vacunación contra la FA en el pasado (ya que la vacuna no ha cambiado en los últimos 70 años). De acuerdo con expertos internacionales en Medicina del Viajero, la recomendación de la OMS aplica a la mayoría de los viajeros, pero ciertos grupos podrían beneficiarse de una revacunación después de 10 años $^{4,5}$. Qué viajeros podrían necesitar este refuerzo es aún controvertido (en la Tabla 2 se describen los posibles factores que favorecen una revacunación). La evaluación de una revacunación en un turista que viaja a Brasil y ha recibido la vacuna contra la FA hace más de 10 años debería realizarla un experto en Medicina del Viajero.

\section{- ¿Qué hacer si el viajero se presenta menos de 10 días antes del viaje?}

La vacuna contra la FA debe ser administrada al menos 10 días antes de entrar a una zona de riesgo (ver más arriba). Si un viajero se presenta con menos de 10 días de anticipación y el viaje no puede ser pospuesto, debería recibir la vacuna, pero debe ser advertido que su protección es incompleta y que las medidas de protección de picaduras de mosquitos son cruciales.

- ¿Es útil medir anticuerpos contra FA para ver si un paciente ha sido expuesto en viajes previos?

No, esta medida no se recomienda. Aunque un viajero haya sido expuesto al virus de la FA previamente, debe igualmente ser vacunado si viaja a un área de riesgo.

\section{- ¿Cómo administrar la vacuna contra la FA} junto a la vacuna tresvírica u otras vacunas?

El Ministerio de Salud de Chile recomienda que los siguientes chilenos reciban la vacuna tresvírica (sarampión-parotiditis-rubéola) antes de un viaje internacional: aquellos nacidos entre 1971 y 1981, niños con solo una dosis de vacuna tresvírica (entre 13 meses y 7 años de edad), y niños no vacunados que tengan entre 6 y 12 meses de edad. Los viajeros que requieren ambas vacunas deben recibirlas con más de 30 días de separación o el mismo día. Si ambas opciones existen, la vacunación separada es preferible, para prevenir posibles interferencias ${ }^{6}$. Las vacunas inactivadas como hepatitis A y B, fiebre tifoidea, influenza, tétanos/difteria/pertussis o antirrábica pueden ser administradas el mismo día o en cualquier momento o con cualquier intervalo con la vacuna contra la FA.

\section{- ¿Qué hacer si un viajero a Brasil ha recibido recientemente la vacuna tresvírica?}

No existe una respuesta estándar para esta pregunta. La co-administración de vacuna contra la FA y tresvírica
Tabla 2. Factores a favor de un refuerzo de vacuna contra fiebre amarilla (después de 10 años o más precoz)

\begin{tabular}{lc}
\hline Factor & Referencias \\
Embarazo (al momento de la primera vacunación) & 4,8 \\
\hline Infección por VIH (al momento de la primera vacunación) & $4,8,9,10$ \\
Cualquier inmunodeficiencia (al momento de la primera vacunación) & 8 \\
\hline Receptor de trasplante de precursores hematopoyéticos & 4,8 \\
\hline Permanencia en una región con un brote activo de FA & 4 \\
\hline Alto riesgo de FA debido a actividades, temporada del viaje o largo período de & \\
estadía en un área endémica & 4,9 \\
Funcionarios de laboratorios que manejan virus de FA & 4 \\
Vacunación con la cepa brasilera (17DD-YF) & 11 \\
Vacunación a edad < 2 años & $8,9,10$ \\
Vacuna contra la FA administrada junto a tresvírica & 6
\end{tabular}

sin 30 días de separación es segura, pero podría afectar la respuesta inmune a alguna de las dos vacunas ${ }^{6}$. Los viajeros que necesitan ser vacunados contra la FA pero recibieron la vacuna tresvírica dentro de los 30 días previos deben ser evaluados y aconsejados por un experto en Medicina del Viajero. Lo mismo aplica si un paciente ha recibido la vacuna contra la FA y tiene una indicación de refuerzo de tresvírica sin tiempo para separar ambas vacunas por 30 días.

\section{- ¿Cómo saber si la FA está presente en una determinada área en Brasil?}

La situación epidemiológica de la FA en Brasil (y en otros países) ha sido muy dinámica en los últimos años. Los médicos que indican la vacuna contra la FA deben revisar regularmente la información epidemiológica actualizada usando fuentes confiables en Internet (Tabla 3). Fuentes informales, ej. grupos de internet o personal de salud no experto, no deben ser consultados. Se puede anticipar que en los próximos años o décadas las regiones costeras de Brasil, actualmente afectadas (incluyendo importantes áreas turísticas) se mantendrán como áreas de riesgo de la FA.

Tabla 3. Vínculos en internet con información epidemiológica en relación a fiebre amarilla

- Centers for Disease Control and Prevention. Yellow Fever Maps. Disponible en: https://www.cdc.gov/yellowfever/maps/index.html

- PAHO/WHO. Yellow Fever: Epidemiological Alerts and Updates. Disponible en: https://www.paho.org/hq/index.php?option=com_topics\&view=rdmore\&cid $=2194 \&$ lemid $=40784 \&$ lang $=$ en

- European Centre for Disease Prevention and Control. Yellow fever. Disponible en: https://ecdc.europa.eu/en/yellow-fever 


\section{- ¿Qué es una dosis fraccionada de vacuna contra la FA?}

La dosis fraccionada es una medida de emergencia que ayuda a vacunar más personas en situaciones de stock limitado de la vacuna. De acuerdo a los expertos, el uso de una dosis fraccionada (un quinto) protege por al menos 12 meses y puede ser usado para individuos $>2$ años de edad en emergencias como un brote. Algunos países (p. ej.: Canadá) han usado esta medida en tiempos de acceso limitado a vacuna para viajeros? ${ }^{7}$. La decisión de ofrecer dosis fraccionada de vacuna contra la FA en situaciones determinadas, depende de directrices de las autoridades de salud y la disposición y capacidad de los centros de vacunación, y no de la decisión personal de un médico. Las precauciones y las contraindicaciones de esta modalidad de administración son las mismas que las establecidas para la dosis completa. No se emite el certificado internacional de vacunación a las personas que reciben una dosis fraccionada de la vacuna.

\section{- ¿Qué hacer si la vacuna de FA no está dis- ponible?}

No hay una respuesta estándar para esta pregunta. Si la vacuna no está disponible y el viaje no se puede posponer o cambiar, el viajero debe solicitar recomendaciones de un experto en Medicina del Viajero respecto a los riesgos individuales y las opciones para reducir estos riesgos.

- ¿Se podrían beneficiar aquellos viajeros a Brasil que no pueden recibir la vacuna de recibir una carta de excepción ("waiver letter")?

No, la carta o certificado que exime de la administración de la vacuna (waiver letter) son utilizadas para via- jeros con contraindicaciones o precauciones a la vacuna, que visitan países, generalmente sin riesgo de FA, que por normativa requieren exhibir un certificado de vacunación al entrar a su territorio. Brasil no tiene este requerimiento. Pero, como se menciona arriba, los viajeros que no estén vacunados deben evitar visitar áreas de riesgo en Brasil.

\section{Conclusiones}

La vacuna contra la FA puede ser indicada por médicos sin experiencia en Medicina del Viajero a turistas que vayan a visitar áreas de riesgo en Brasil, pero deben evaluar cuidadosamente a cada uno de ellos en relación a las vacunas que han recibido recientemente, edad, embarazo, medicamentos y antecedentes de enfermedades.

En las siguientes situaciones el viajero debe ser referido a un experto en Medicina del Viajero:

- Si el paciente tiene condiciones que contraindiquen la vacuna o en aquellas en que hay que tener precauciones especiales (Tabla 1)

- Si el paciente recibió la vacuna hace más de 10 años y debe evaluarse la conveniencia de revacunar (Tabla 2).

- Si las vacunas de FA y tresvírica están recomendadas y no pueden ser administradas el mismo día ni tampoco separadas por más de 30 días.

\section{Resumen}

La aparición de fiebre amarilla en las costas brasileñas, lugares de alto interés turístico, ha provocado una alta demanda de vacunación. Este articulo entrega respuestas a las principales consultas sobre la vacuna de fiebre amarilla y puede servir como guía para médicos no expertos en Medicina del Viajero.

\section{Referencias bibliográficas}

1.- Hamer D H, Angelo K, Caumes E, van Genderen P J J, Florescu S A, Popescu C P, et al. Fatal yellow fever in travelers to Brazil, 2018. MMWR Morb Mortal Wkly Rep 2018; 67: 340 .

2.- $\quad$ Monath T P, Gershman M, Staples J E, Barrett A D T. Yellow fever vaccine. In: Plotkin SA, Orenstein WA, Offitt PA, editors. Vaccines. $6^{\text {th }}$ ed. Edinburgh: Elsevier/Saunders; 2013. Pp. 870-968.

3.- WHO. Vaccines and vaccination against yellow fever: WHO Position Paper, June 2013-recommendations. Vaccine 2015; 33: 76-7.

4.- Staples J E, Bocchini JA Jr, Rubin L, Fischer $M$, Centers for Disease Control and Prevention (CDC) Yellow Fever Vaccine Booster Doses: Recommendations of the Advisory Committee on Immunization Practices, 2015. MMWR Morb Mortal Wkly Rep 2015; 64: 647.

5.- Patel D, Simons H. Yellow fever vaccination: is one dose always enough? Travel Med Infect Dis 2013; 11: 266-73.

6.- Nascimento Silva JR, Camacho LA, Siqueira MM, Freire Mde S, Castro YP, Maia Mde L, et al; Collaborative Group for the Study of Yellow Fever Vaccines. Mutual interference on the immune response to yellow fever vaccine and a combined vaccine against measles, mumps and rubella. Vaccine 2011; 29 : 6327-34.

7.- Yellow Fever Working Group; Committee to Advise on Tropical Medicine and; Travel (CATMAT). Interim Canadian recommendations for the use of a fractional dose of yellow fever vaccine during a vaccine shortage. Can Commun Dis Rep 2016; 42: 15860 .
8.- Public Health England. The Green Book. Chapter 35: Yellow Fever (June 2018). Available at: https://assets.publishing.service. gov.uk/government/uploads/system/uploads/ attachment_data/file/716247/Greenbook_ chapter 35 v2 Yellow Fever.pdf

9.- Collaborative group for studies on yellow fever vaccines. Duration of post-vaccination immunity against yellow fever in adults. Vaccine 2014; 32: 4977-84.

10.- Amanna I J, Slifka M K. Questions regarding the safety and duration of immunity following live yellow fever vaccination. Expert Rev Vaccines 2016; 15: 1519-33

11.- Campi-Azevedo A C, Costa-Pereira C, Antonelli L R, Fonseca C T, Teixeira-Carvalho A, Villela-Rezende $\mathrm{G}$, et al. Booster dose after 10 years is recommended following 17DD-YF primary vaccination. Hum Vaccin Immunother 2016; 12: 491-502. 